\title{
Article
}

\section{Personality disorder co-morbidity in primary care 'Improving Access to Psychological Therapy' (IAPT) services: a qualitative study exploring patient perspectives on treatment experience}

Lamph, Gary, Baker, J, Dickinson, T and Lovell, K Available at http://clok.uclan.ac.uk/34325/

Lamph, Gary ORCID: 0000-0002-4099-2812, Baker, J, Dickinson, T and Lovell, $K$ (2020) Personality disorder co-morbidity in primary care 'Improving Access to Psychological Therapy' (IAPT) services: a qualitative study exploring patient perspectives on treatment experience. Behavioural and Cognitive Psychotherapy . ISSN 1352-4658

It is advisable to refer to the publisher's version if you intend to cite from the work. http://dx.doi.org/10.1017/S1352465820000594

For more information about UCLan's research in this area go to http://www.uclan.ac.uk/researchgroups/ and search for < name of research Group>.

For information about Research generally at UCLan please go to http://www.uclan.ac.uk/research/

All outputs in CLoK are protected by Intellectual Property Rights law, including Copyright law. Copyright, IPR and Moral Rights for the works on this site are retained by the individual authors and/or other copyright owners. Terms and conditions for use of this material are defined in the policies page. 


\section{Personality disorder co-morbidity in primary care 'Improving Access to Psychological Therapy' (IAPT) Services: A qualitative study exploring patient perspectives on treatment experience.}

Background: High numbers of people present with common mental health disorders and co-morbid personality disorder traits in primary care 'Improving Access to Psychological Therapies' (IAPT) services in England and they receive suboptimal treatments. No previous studies have explored the treatment experiences or needs of this patient population in England.

Aims: This qualitative study explored the treatment experiences of patients $(\mathrm{N}=22)$ with common mental health difficulties and co-morbid personality disorder as indicated by a score of 3 or more on the 'Standardised Assessment of Personality Abbreviated Scale' (SAPAS) in receipt of primary care based IAPT treatment.

Method: A qualitative health research approach was used. Qualitative individual face-to-face semi-structured interviews were conducted. All interviews were audio recorded, data was transcribed verbatim and analysed using a framework analysis approach.

Results: Findings revealed a need to adapt away from prescriptive cognitive behavioral therapy (CBT) treatment models towards more flexible, personalised and individualised treatment with this patient group. Time to emotionally offload, build a therapeutic relationship and link past experiences to presenting problems were highlighted as important.

Conclusions: For the first time the needs and treatment experiences of this patient group have been explored. This paper provides a unique patient experience insight that should be considered when exploring new approaches to working with and developing effective interventions via a stepped care approach.

\section{Keywords:}

Personality Disorder, Primary Care, Improving Access to Psychological Therapies (IAPT)

\section{Introduction}


In 2008, an ambitious initiative to make psychological therapies more accessible to the general-public in England who present with common mental health disorders such as anxiety and depression was developed called 'Improving Access to Psychological Therapies Services' (IAPT). IAPT provides a stepped care model, in which low dose cost effective psychological treatments are available. IAPT services are situated in primary care, outside of specialist mental health service provision (Department of Health [DOH], 2008). Personality disorder is often difficult to identify and overlooked due to the high comorbidity of conditions such as anxiety and depression, that are often viewed as separate problems ( $\mathrm{DOH}, 2009)$. In a recent study of IAPT patient populations that focused on the prevalence of co-morbidities, $69 \%$ of patients were reported to present with a high risk of personality disorder (Hepgul et al., 2016). People who present to IAPT with co-morbid personality disorder traits, on the basis of screening positively (score of 3 or more, which is indicative of personality disorder diagnosis) on the 'Standardised Assessment of Personality - Abbreviated Scale (SAPAS) (Moran et al., 2003), are also known to have poorer treatment outcomes (Goddard, Wingrove \& Moran., 2015). Personality disorder traits are difficulties and characteristics of personality disorder, but do not necessarily constitute a diagnosis (Livesley, Lang \& Vernon, 1998). Personality disorder is a stigmatising and contested label and hence in undiagnosed populations should be approached with sensitivity (Anonymous for review., 2019). The UK's National Institute for Health and Clinical Excellence (NICE) guidelines suggested that only longer-term treatments should be offered to people with personality disorder (NICE, 2009). There is a lack of research and evidence-based shorter-term interventions made available to this patient group (Paris, 2013). The evidence base for longer term interventions verses shorter term interventions is however inconclusive, under researched and by sticking rigidly to this only causes a bottle neck effect and increased waiting lists (Paris, 2017). In recent years attention has been paid to this deficit in both the UK (Crawford et al., 2018) and internationally (Laporte, Paris, Bergevin, Fraser \& Cardin., 2018). The is a need for short term interventions being made more readily available and a stepped care approach that is available for most other mental health conditions being supported for people with personality disorder (Crawford et al., 2018; DOH, 2011; Laporte et al., 2018; Mind, 2018). It has subsequently been suggested that routine screening and new approaches to working with this patient group should be adopted in IAPT services 
(Goddard et al., 2015). However, there is little clarification of what these adaptions should look like, or of any consultation with patients to inform the development of such interventions. The Royal College of Psychiatrists (RCPsych) released a position statement (RCPsych, 2020) in which reference is made to having stepped care and tiered treatment approaches to personality disorder treatment and support was acknowledged in line with the ICD-11 changes. Levels of personality disorder severity are described within the ICD-11 (WHO, 2019) as mild, moderate and severe. There is a gap in the research evidence-base for interventions directed at mild - moderate severity, or in the provision of time-limited interventions for people with personality disorder in primary care. Research interviews have been conducted with IAPT healthcare professionals to understand the perspectives of their views of working with people with co-morbid personality disorder in primary care services (Lamph et al., 2019). However, the needs and perspectives of this patient group have yet to be explored. In current literature their needs are discussed from the perspectives of expert opinion, not the patients themselves (DOH, 2011; Livesley, 2005; Moran \& Crawford., 2013; Tyrer and Duggan., 2007). This study addresses this deficit in the literature by providing the first study that explores needs and treatment experiences in IAPT directly from the patient perspectives.

$\underline{\text { Aims }}$

This study explored the treatment experience of patient participants who present to IAPT services for treatment with common mental health disorders and are identified to additionally present with co-morbid personality disorder traits.

\section{Methods}

\section{Study Design}

A qualitative health research approach was adopted to explore the research aim. Individual qualitative semi-structured interviews were conducted with patients who had common mental health difficulties (including anxiety and depression) and identified co-morbid personality disorder traits. Data was transcribed verbatim and inputted into a data analysis software package QSR NVivo10 @. Analysis was conducted using a framework analysis approach (Ritchie, Lewis, McNaughton- 
Nicholls and Ormston, 2014). An inter-related study exploring the perspectives of IAPT healthcare professionals responsible for delivering therapies to this patient group has also been conducted, the results can be viewed separately (Anonymised for review, 2019). A favourable ethical opinion via proportionate review was received in May 2015 by NRES Committees North of Scotland (Rec Reference: 15/NS/0043).

\section{Lived Experience Research Advisory Group}

The lived experience advisory group was recruited from a local NHS Trust involvement scheme. This group was made up of six people who had lived experience of personality disorder and whom had also received previous psychological therapy. Recruitment flyers were developed by the group, ensuring that the development of research materials was user friendly and sensitively developed using non-stigmatising and understandable language. The research advisory group chose to take a descriptive approach in the development of research materials, to ensure that we did not label people with a diagnostic label who had not received a diagnosis. Instead the term 'understanding long standing emotional difficulties' was chosen as the title for the recruitment materials, with a descriptive breakdown of common personality disorder traits / difficulties. This group met on 6 separate occasions to support the development of the research materials and the recruitment strategy.

\section{$\underline{\text { Recruitment Processes }}$}

Recruitment flyers were placed in waiting rooms but also given to those in receipt of or had recently engaged in an IAPT intervention. The flyers were designed to highlight personality disorder traits in understandable and descriptive language and invited patients who could relate to the descriptors, to express interest in this research. An IAPT intervention was defined as those in receipt of a Step 2 which is defined as a 'low intensity intervention' (up to $81 / 2$ hour sessions self-help model) or Step 3 treatments 'high intensity intervention' (up to 20 1-hour sessions of cognitive behavioural therapy) (Richards et al., 2012). Potential participants were given the option to make direct contact with the research team to express interest in becoming involved or via their therapists. Those expressing interest were then invited to take part in a telephone screening interview to determine their eligibility for the study. 


\section{Study Sample}

Participants were recruited from a single site IAPT service using a purposive sampling approach. Suitability for participation in the study was then determined via a telephone screening interview with lead author (Anonymised initials to be added). Inclusion Criteria:

- Participants were required to be in receipt of IAPT treatment or to have received IAPT treatment within the past 12 months.

- A Score of 3 or more on the SAPAS

- A minimum of six weeks into the treatment timeframe to ensure adequate exposure to treatment had been offered.

- A minimum of one treatment session had to have been attended for inclusion hence those who prematurely discontinued treatment were not excluded.

- Participants were age 18 or over.

Exclusion Criteria:

- The presence of significant language barriers that required a translator.

- Marked acute impairment, such as florid psychosis, actively suicidal, learning disability / organic illness or substance misuse dependency.

- Referred patients who did not commence IAPT treatment.

To ensure patients with common mental health difficulties and co-morbid personality disorder traits were recruited we adopted the use of the SAPAS (Moran et al., 2003). Whilst this is not a diagnostic tool, it has during a preliminary validation been tested in its accuracy to identify personality disorder with a score of 3 out of 8 possible questions being indicative of a DSM IV (American Psychiatric Association (APA), 2000 ) diagnosable personality disorder in $90 \%$ of cases. We therefore employed a score of 3 or above for inclusion into the study. Furthermore, the SAPAS has been deemed feasible for usage in routine clinical practice for the identification of people with personality disorder (Moran et al., 2003) and used in the identification of people with personality disorder co-morbidity in IAPT (Goddard et al., 2015; Grant et al., 2014; Hepgul et al., 2016). Those who met criteria following the screening were sent a detailed participant involvement form. Recruitment of participants was closed 
once the research team had agreed saturation had been reached and no new themes were identified.

\section{Data Collection}

All interviews were conducted face to face by researcher (Anonymised initials) with a total time $28 \mathrm{~h}: 31 \mathrm{~m}: 31 \mathrm{~s}$ captured on a encrypted digital recording device, the 'mean' interview duration was $1 \mathrm{~h}: 28 \mathrm{~m}$. Participants were given a choice of venues with all opting to be interviewed within IAPT clinics or NHS buildings, however all were given the option of suggesting alternative venues including university buildings. The topic guide was developed over several face to face meetings with the lived experience research advisory group and (anonymised researcher initials to be added). This was a collaborative effort in which areas for inclusion were agreed following much discussion. The topic guide covered 5 key areas for exploration of: 1) the patient's background and reasons for contact with the IAPT service 2) the patient's perspectives relating to their needs and difficulties 3 ) the patient treatment experiences in IAPT 4) endings in therapy. An additional point was included, if it applied to the participant 5) reasons for therapy drop out. After each session a field note journal was kept capturing any non-verbal observations that would go unnoticed on the audio recording.

\section{Data Analysis}

All verbatim data was inputted into NVivo and a framework analysis approach (Ritchie et al., 2014). Framework analysis allowed for the inclusion of deductive themes, which shaped the initial framework and the emergence of inductive themes that came from the emerging research data that was collected (Gale, Heath, Cameron, Rashid \& Redwood., 2013). The NVivo framework analysis function was used to develop and populate the framework, hence providing a secure electronic data management tool. However, it should be noted that the analysis was performed by the researchers. The lead author (Anonymised, initials to be added) initially coded and developed the emerging themes however co-authors (Anonymised Initials to be added) verified the finalisation and reaching a consensus on the themes reported here. The framework approach is a systematic approach that ensures good 
auditability of the data (Gale et al., 2013). The framework matrix that is developed using this approach provides the opportunity to perform both 'within case' and 'across case' analysis of the data which makes managing and interpreting large data sets more manageable. Further detail can be viewed in the University of Manchester Repository where the PhD thesis that this study was part of is held (Anonymised for review).

\section{Results}

Thirty-three participants expressed interest in being involved. Of these twenty-four were eligible using the SAPAS criteria, two declined interviews. Eight did not meet the inclusion criteria and one withdrew interest before being screened. Twenty-two were interviewed. Participants were evenly divided between those in receipt of, or recently discharged from Step 2 and Step 3 treatments and recruitment was discontinued once data saturation had been determined. Three participants were in the process of being escalated from Step 2 to Step 3 treatment. Most participants were female $(\mathrm{N}=13)$ and 'White British' $(\mathrm{N}=21)$. Scores on the SAPAS ranged from 4-8 with a mean score of 6 .

\section{Attach table 1 here? (Author Suggestion)}

\section{Findings}

Four key themes emerged from the data: 1) The IAPT Journey, 2) One size fits all, 3) Common needs and 4) Opposing the 'one size fits all' experience.

\section{The IAPT Journey}

This theme related to participants experience of the IAPT Journey, which focussed upon the journey to accessing the service and then the operational processes participants reported. For many participants a build-up of problems and deterioration in mental health preceded them seeking support and their subsequent referral to IAPT services. Initial contact with services was consistently viewed as difficult. A quarter of the participants described problems as having a significant and detrimental impact on their lives and functioning. 
"I'd been feeling like crap for about four months and then one day it just got too much, and I burnt myself with my straighteners. Then that scared me, so I went to the doctor" (P9, Step 2).

When seen in IAPT, participants frequently described a lack of treatment choice. They reported rarely being involved in treatment decisions and raised concerns that therapy was delivered in a manualised prescriptive way.

"They probably use the same strategy on the next person, the next person and the next person. It doesn't mean it's going to work on all of us. We're all different individuals" (P18 Step 2).

Participants described that the number of sessions offered was driven by service constraints rather than defined by individual needs:

"It's like putting a price tag on it [Number of sessions]. It just feels as though you're allocated so much money, once your money's run out there's the door, see you. You're left on your own... And I know that it's not the therapists fault, it's the system. I think the system's totally wrong. I think it should be analysed and treated on an individual case instead of on an average... I mean, who can be average when they've got mental health issues? They can't, can they? And I think that is a very big let down!" (P20, Step 3).

Cognitive behavioural therapy was commonly reported as the core treatment in IAPT, a small minority reported being offered a different choice of treatment. Additionally, some participants described having to go through the process of steps even when it was recognised that Step 2 treatments were not working.

"You've got to start somewhere, but I suppose if after the first couple of sessions you would understand that it's not really going to... In other words, it's not flowing, it's grating, it's going against the grain with me... What's the point? It's just a waste of weeks" (P2, Step 2).

Whilst positive and negative views were reported from treatment experience at both steps, a majority of those receiving Step 2 treatments were negative.

"She'd give me a piece of paper to write things down on every day and other stuff and I thought it was a bit pointless..." (P18, Step 2). 
Participants receiving Step 3 treatments were more positive due to increased flexibility, therapist skill, duration of session and choices of treatment offered. Those with mixed views of Step 3 revealed that therapy was either too difficult without the added component of additional social support alongside therapy or as lacking the depth required to understand a complexity of their problems.

\section{One size fits all}

A key theme was identified relating to the rigidity of treatment / interventions offered in IAPT and the comparisons made across the different steps. Those receiving Step 2 treatment described more negative experiences of manualised approaches to treatment than those who had received Step 3 intervention.

'You don't feel engaged by it, because you feel that you're not necessarily being listened to and what your individual issues are, because although there are common Step 2's and common behaviours that people might show, you're an individual at the end of the day and your problems and your reactions and your emotions are not necessarily the same as anybody else's. And I don't think when it's, right, this week we're doing that, next week we're doing that, you feel like you're not fully engaged, and you almost feel right, well, let's just go along and get to the end of the therapy" (P16 Step 2).

Furthermore, time pressures experienced at Step 2 were also reported counterproductively.

"I think because it just feels like you're rushed. It's like you've sat down and then got back up to get out... Really you're getting settled down for 15, 20 minutes, might have kicked into, yeah, I can see where you're coming from, and then it starts getting the ball rolling, and then as soon as the momentum's started it's like right, l'll see you next week" (P2, Step 2).

Rigid treatments at Step 2 were commonly reported as; 'mechanistic, scripted, shallow, robotic, going through the motions, lack of flexibility, here and now only focus' shared. This is illustrated by a participant account.

"People like me with long term mental illness can't solve anything in six sessions... I just seem to get the same answers to the questions over and over and over again. I don't seem to get anything different" (P18, Step 2). 
Others described the therapist approach as being important "I think there's been variable quality to the therapy, depending on who you've seen. I've had sessions with some therapists and you think wow! That was good, they're really insightful. Then others that seem a bit more mechanistic, almost as though they're going through the motions of the therapy rather than fully engaging with it" (P16 Step 2).

Some participants felt that negative treatment experience could result in drop out and /or and become a barrier to future treatment seeking. One participant described how her therapy experience was focussed around one breathing technique and that this has made her reluctant to seek further treatment for fear that she will be offered the same treatment again

“...I'm wary for the future, because I think if I need to go back, would I actually benefit? Or am I just going to go through the whole [technique] thing again" (P17, Step 2).

Not all Step 2 experiences were negative. One of the participants described how for the first time in 30 years she had felt listened to and stepped up into a treatment that could help her.

'Yes, even though he'd said numerous times we're not going to leave you this time, I still didn't believe him, because l'd been told l'd get help before and it's never...you know, but he was insistent and he was like, you're not being left this time, there's going to be somebody there for you, so that was like, oh my god. I didn't quite know what to do with it. A simultaneous feeling of fear and relief, obviously because there's a lot of stuff I've got to go through now and talk about, but at the same time relief that actually somebody for the first time is actually taking me seriously and listened and said you need help, we'll get it for you" (P10, Step 2).

Step 3 participants would more commonly describe positive or mixed experiences. The time to be listened too at Step 3 was deemed to be important to participants, this additional time, flexibility and depth to therapy was seen as the most helpful element of treatment. When flexibility and choice was experienced this led to positive patient feedback.

Alternative therapies to CBT were rarely offered, only 2 participants made specific reference to different approaches, with mindfulness and acceptance and 
commitment therapy (ACT) being described as the provided IAPT treatments. Negative views were rarely expressed by participants who had received a Step 3 treatment.

Participants who were trying to make sense of how their early life experiences had impacted on their presenting difficulties felt that they meet with a dismissive stance.

"I think my issue with CBT and, I know, you're completely going against what it actually is, it completely ignores anything from the past, I know it's just changing your way of thinking, but I couldn't come to terms with the things that had caused my low mood and because I wasn't having the opportunity to talk about them properly, I didn't feel like I was able to get past it” (P21, Step 2).

Participants conveyed how the rigidity of treatments and the adoption of a "one size fits all" (P6, Step 2) approach had a counterproductive impact on their recovery. Premature endings to therapy are reported with one participant describing almost reaching a stage of recovery, only for the therapy to end as she had reached her maximum allotted sessions following a full course of treatment at Step 3.

\section{$\underline{\text { Common needs }}$}

This theme focussed on the perspectives of participant needs in relation to their presenting problems. Participants described difficulty in articulating what their needs were and instead would often discuss their needs in the context of problem areas they wished to address in therapy. Interestingly, all participants described problems in forming and maintaining relationships, interpersonal effectiveness difficulties and a majority reported difficulties in managing their emotions. The relationship difficulties highlighted by participants included a lack of trust of others which led to an avoidance of social interaction and forming relationships with others. Attachment and abandonment childhood experiences were raised by several participants.

Participants with these experiences expressed how they would often push away relationships through fear of rejection. This is illustrated in the following example: "Well, like in relationships, messing relationships up, horribly; walking away from people who care about me; cutting people out of my life... And I'm suspecting now that perhaps I sabotage my relationships as a way of avoiding being let down by 
people, because if I can push them away first then they can't let me down and hurt me" (P4, Step 3).

Just under half of the participants described themselves as socially withdrawn and guarded.

"You see I don't know whether to be myself because if I be myself I risk people genuinely not liking me as a person. If I put the walls up and I am somebody else then I risk never knowing if anybody is going to like me as a person, I risk never making any real friends because they are making friends with someone who doesn't really exist" (P1, Step 3).

A lack of confidence with interpersonal effectiveness and emotional regulation was consistently described as were low self-esteem and poor self-image. A quarter of participants felt that their problems were related to an inability to manage everyday life stressors.

"It's being able to function in day to day life, really. I can't" (P13, Step 3).

Six participants described being suicidal or as having experienced long standing suicidal ideas throughout their lives. Just under half described using the following coping strategies to deal with their emotions: self-injury, alcohol, illicit substance or painkiller abuse.

"There is no way of sugar coating it, I was suicidal. I was at the point of no return really" (P1, Step 3).

Analysis of data indicated that needs were unique to the individual with a variety of views and opinions shared. Due to difficulties articulating needs, participants instead chose to describe difficulties that were either 'assisted' which could be referred to as 'met needs' or 'unaddressed difficulties' which could be referred to as 'unmet needs'. Participants however rarely reported all needs being met. The most frequently reported met need was the opportunity to talk, be listened to and the opportunity to offload.

"Well, the actual talking to somebody is the biggest thing. Irrespective of what all the techniques have shown, the fact that it's almost unloading the burden and saying this is what's going on in my life, this is why I'm feeling..." (P16, Step 2). 
Some common and routine CBT approaches were described by participants as beneficial, however, participants were often were wanting help with managing everyday life challenges, overcoming interpersonal difficulties and managing difficulties in relationships rather than receiving a defined intervention such as anxiety management. This lack of individualised focus was attributed to the lack of space and time to offload within IAPT treatments.

When treatment failed to include their past, participants felt misunderstood and concerned that therapy would lack the capability of reaching their core problems, leaving participants feeling frustrated, especially those who had specifically asked to discuss past experiences and the impact on their current problems. Only a few participants felt able to discuss the past and present within IAPT therapy.

Participants felt constrained by the rigidity of the therapist to always return the focus to anxiety or depression rather than exploration of the wider picture.

\section{Opposing the 'One size fits' all' experience}

This theme outlines the factors that mattered most to the participants and provides a contrasting perspective to the 'one size fits all' experience. Therapeutic connections, personalisation and therapist flexibility were all highlighted to be of importance to future treatment delivery.

The connection participants had with their therapist was overwhelmingly deemed to be of high importance.

"I think it's having somebody there who knows how to get you over this. Having contact with somebody that you feel safe with, basically, and they can say to you look, I'm here and I'll make you better, we'll work through this together" (P20, Step 3).

Connection with the therapist is something participants described as being formed early. Early negative connections with the therapist were described as being difficult to recover from.

“Didn't feel right from the start, felt cold, when you're talking to a person or psychiatrist you want them to talk to you like a person, like, you wanting to understand my life and try and help me to understand my own life... just there's nothing there between me and her, like a connection. You need a connection to 
work with anybody really and like I say, it was like there was no connection between me and her" (P18, Step 2).

A combination of unique and individual factors enabled participants to connect effectively with their therapists. Participants provided a range of different combinations of factors they felt were important in developing such connections. Identified factors included: trust, honesty, humour, personalised treatment, mutual respect, shared interests, compassion, empathy, validation, not feeling judged, interpersonal effectiveness of the therapist. However, no specific combination of factors, were consistently provided, instead they were unique to the individual participants and their preferences.

Personalisation of treatments were associated with the participant perception that the therapist knows them as an individual. Participants judged this therapist knowledge based on the therapist ability to recall personal facts about them, such as names of family, friends and pets, events, hobbies or making reference to previous session discussions. This recall and feedback was seen to be demonstration of the therapist attention and interest in the patient, for example one participant recalled:

"When I was talking about all these friends and stuff, he remembered all the names of people I was talking to. This made a massive difference, he remembered, it's important to him, it's the little things ...To me it meant that he'd listened, he'd took it on board and we could pick up from where we were without having to go back over stuff" (P22, Step 3).

Conversely participants would often report a process in therapy that felt impersonal and robotic. Therapist training and the rigidity of the IAPT system were identified as possible factors that were barriers to personalised approaches.

"You know you spend half the sessions going over the same things you have already gone over, and it's very frustrating, you know and it's like I have already told you about this, even if they just look through the notes 5 minutes before you come in and refresh themselves, so they are not confusing you with their other patients. Which you know, I understand as a human being you are going to make mistakes, but it's just if you go in there and you think this person doesn't know me at all we are just going through a robotic stage here" (P3 step 2). 
The need for therapist flexibility and moving away from manualised approaches to work directly with patient difficulties was reported by participants. Those receiving a Step 3 treatment were more likely to report personalised and flexible experiences than those at Step 2, who more commonly described mechanistic and scripted experiences. Flexibility of approach was not just related by participants to the treatment they received, but also to therapist understanding of missed appointments, time keeping etc. and the display of an empathetic and understanding stance towards other life factors that sometimes get in the way of therapy attendance and engagement.

"He was very flexible. Many times I had to cancel due to work - because I do work full time - so I had to cancel, say, two sessions due to work. He was very understanding" (P14, Step 3).

\section{Discussion}

Personality Disorder co-morbidity is highly prevalent in IAPT services and indicative of sub-optimal treatment outcomes (Goddard et al., 2015). Currently there are no personality disorder specific evidence-based treatments routinely available to this patient population in England. This paper provides for the first time an insight into the patient perspectives of using IAPT services.

A key challenge when working with this patient group is moving away from manualised 'one size fits all' approaches, that are fuelled by the evidence-based treatment protocols for CBT and constraints of the service, which participants often described as being rigid and impersonal treatment approaches. The identified needs of the participants in this study and their presentations was not dissimilar from patients who present to secondary mental health services with diagnosed personality disorder (Sampson, McCubbin \& Tyrer, 2006). The research team who have expertise in both primary and secondary services felt that whilst many of the characteristics were similar to patients with personality disorder traits and diagnosis in secondary service, they presented equally with high levels of complexity but with lower levels of risk. Many of the participants reported identifiable personality disorder traits, which were described as key difficulties that were being unresponsive to standard CBT treatments. The reporting from participants of maladaptive coping strategies to deals with everyday life stressors including self-injury, alcohol and 
substance misuse difficulties were frequently described. The presenting needs were deemed to be unique to the individual as was their experience and the self-reported effectiveness of their treatment.

Participants were largely negative if in receipt of a Step 2 treatment, describing the rigidity of treatment approaches and lack of time to develop relationships with the therapist as a barrier to treatment progress. Participants also highlighted the importance of individualised and personalised approaches that commonly were not met at Step 2. Conversely Step 3 treatments were reported with more positivity. The key components that resulted in positive treatment experience of Step 3 included the opportunity for offloading of difficulties, increased session duration / time and flexibility of the therapist. Alternative to CBT approaches or third wave CBT treatments were rarely discussed but when they were they were described with positivity. It was reported that many therapists failed to acknowledge the impact of past experiences on presenting difficulties and therefore developmental formulations may enable greater recognition of this as outlined by Davidson (2002) and hence enhance treatment experience and patient understanding of their presenting difficulties.

The development of an effective therapeutic relationship is of particular importance due to attachment, relational and interpersonal difficulties that commonly present (Davidson, 2002). Participants identified factors that enable the development of a good therapeutic relationship, however these are unique to the individual and whilst awareness of the identified key factors from this research are important to acknowledge there will be cases were therapist and patient relate to each other with ease and other relationships that may clearly be more strained. Flexibility of approach and being treated in a personalised / individualised approach was key to the positive treatment experience. This patient group will frequently encounter general practitioners (GPs) and in a recent qualitative study that explored GPs experiences, it was concluded that primary care IAPT services continue to struggle to meet the needs of this more complex patient group, and hence their needs often go unmet (French, Moran, Wiles, Kessler\& Turner, 2019). Within the UK, there continues to be a postcode lottery as to what service provision is available to this patient group with some primary care IAPT services accepting referrals for people with personality disorder and others refusing (French et al., 2019; Mind, 2018). 
However brief interventions have been adapted from known effective treatment strategies for those presenting with less complexity and as shorter-term interventions with early positive results being reported outside of IAPT services (Crawford et al., 2018; Laporte et al., 2018). Crawford et al (2020) have reported positive findings for their pilot of low intensity interventions in secondary mental health services and have suggested that further attention is required to address short-term primary care approaches. The changes to the ICD-11 (World Health organisation [WHO], 2019) also provides an opportunity to look differently at how we work with those presenting with personality co-morbidity at a lower level of severity and hence provides an opportunity to further develop shorter term interventions for those who present with more milder difficulties but are unresponsive to current treatment provision. Additionally, whilst not all participants disclosed past childhood traumas in this study the importance of linking past to present was frequently highlighted, the growing interest in trauma informed care approaches should be considered if links to past trauma are identified. Key principles for trauma informed approaches are outlined by Sweeney, Clement, Filson \& Kennedy (2016) and include; the need to recognise trauma and re-traumatisation, development of trustworthiness and transparency, collaborative working approaches, empowerment, choice and control, safety, survivor partnerships and clear trauma informed pathways.

\section{Limitations}

A potential limitation of this study was in the single site it had been conducted in hence the results may not be the same as other IAPT services. The comments of the participants in this study who met the criteria for personality co-morbidity may not be limited to those with personality difficulties. IAPT healthcare professionals were tasked with supplying the recruitment flyers to those on their current case-loads. This could have resulted in selection bias on who was asked to participate. Despite trying to mitigate this, with regular updates and discussion with the research team, it is remains unclear as to whether all potentially eligible participants were invited.

A further limitation of this study is in the lack of ethnic diversity. People of ethnic origin are less likely to use IAPT services (Evans, Green, Sharma, Marinho \& Thomas, 2014). However, the area in which this research was conducted is one of the least ethnically diverse areas in the North West of England with only $2.7 \%$ being 
from a Black or Minority Ethnic (BME) backgrounds, hence the sample was representative of the research site (Public Health England, 2019).

Mixed scores on the SAPAS were identified amongst participants who took part. SAPAS can be over inclusive particularly if implemented on presentation to services (Moran et al., 2003). Those with higher SAPAS scores presented with more complexity generally than those with lower scores, however, we did not differentiate or carry out any additional analysis based on complexity of presentation as determined by higher SAPAS scores.

\section{Conclusion}

Personality disorder co-morbidity has in recent years been acknowledged to be highly prevalent in primary care populations and impacts negatively on treatment outcomes. New and novel approaches are required to improve outcomes for people with personality disorder co-morbidity in primary care.

To improve the treatment experience of this patient group, therapists are required to pay more attention to impact that the past may have had on the present, which can be achieved by capturing this via the use of developmental formulation driven approaches. Time and flexibility should be afforded hence allowing the patient to address life difficulties as they arise, hence ensuring a personalised and individualised treatment experience. A focus on interpersonal skills development, management of emotions and problem-solving approaches to life stresses and crises should be adopted. Dialectical Behavioural Therapy skills (Core Mindfulness, Distress Tolerance, Emotional Regulation and Interpersonal Effectiveness) are recommended as they align well to the identified patient needs, with the frequently reported lack of confidence relating to relationships, emotional management and impulsivity reported within this data. When childhood traumas are identified trauma informed care principles should be carefully considered.

This paper provides for the first time a unique exploration into the needs and treatment experiences of people who present to primary care IAPT services with comorbid personality disorder. It provides the necessary preparatory insight for consideration when working with people who present with co-morbid personality disorder in primary care IAPT services. New knowledge relating to the needs and 
treatment experiences of this patient group receiving treatment in IAPT have been explored. The findings have transferable benefits for therapist who can identify patients presenting with often undiagnosed personality disorder co-morbidity and should be considered when exploring new approaches to working with and developing interventions and new approaches to working with this patient group in primary care.

\section{References}

Anonymized for Review (2018) Enhancing understanding of the experience of people with common mental health disorders and co-morbid personality disorder traits who present to primary care IAPT services. PhD Thesis; University of Manchester Institutional Repository ISNI; 0000000476580064.

https://www.research.manchester.ac.uk/portal/en/theses/enhancing-understandingof-the-experience-of-people-with-common-mental-health-disorders-and-comorbidpersonality-disorder-traits-who-present-to-primary-care-iapt-services(95a9bf9d-e814$\underline{48 b c-b 0 e 3-892 f 8 b 6687 d 6) \cdot h t m l}$

Anonymized for Review. (2019) Personality disorder co-morbidity in primary care 'Improving Access to Psychological Therapy' (IAPT) Services; A qualitative study exploring professionals perspectives. Personality and Mental Health. 13 (3). pp. 168179. ISSN 1932-862.

American Psychiatric Association (APA). (2000) Diagnostic and Statistical Manual of Mental Disorders. (DSMIVTR) (4th ed. Text Rev). APA, Washington DC.

Crawford, M, J., Thana, L., Parker, J., Turner, O., Carnet, A., McMurran, M., Moran, P., Weaver, T., Barrett, B., Roberts, S., Claringbold, A., Basset, P., Sanatinia, R. \& Spong, A. (2020) Structured Psychological Support for people with personality disorder: feasibility randomized controlled trial of a low-intensity intervention. BJPsychiatry Open 6, e25, 1-9. doi: 10.1192/bjo.2020.7.

Crawford, M, J., Thana, L., Parker, J., Turner, O., Xing, K, P., McMurran, M., Moran, P., Weaver, T., Barrett, B., Claringbold, A., Basset, P., \& Sanatinia, R. (2018) Psychological Support for Personality (PSP) verses treatment as usual: study protocol for a feasibility randomized controlled trial of a low intensity intervention for people with personality disorder. Trials. 19: 547. https://doi.org./10.1186/s13063018-2920-0. 
Davidson, K. (2002) Cognitive Therapy for Personality Disorders. Arnold: London.

Department of Health (DOH). (2008) Improving Access to Psychological Therapies Implementation Plan: National guidelines for regional delivery. Crown Copyright: London.

Department of Health (DOH). (2009) Recognising Complexity: Commissioning guidance for personality disorder services. Crown Copyright: London.

Department of Health (DOH). (2011) Talking Therapies: A four-year plan of action. A supporting document to No Health Without Mental Health: A cross government mental health outcomes strategy for people of all ages. Crown Copyright: London.

Evans, L., Green, S., Sharma, K., Marinho, F \& Thomas, P. (2014) Improving access to primary mental health services: are link workers the answer? Journal of Primary Care. 6 (2) 23-28.

French, L., Moran, P., Wiles, N., Kessler, D. \& Turner, K, M. (2019) GP's views and experiences of managing patients with personality disorder: A qualitative interview study. British Medical Journal Open. 9:e026616. Doi:10.1136/bmjopen2018-026616.

Gale, N, K., Heath, G., Cameron, E., Rashid, S. \& Redwood, S. (2013) Using the framework method for the analysis of qualitative data in multi-disciplinary health research. BMC Medical Research Methodology. 13, 117.

Goddard, E., Wingrove, J \& Moran, P. (2015) The impact of comorbid personality difficulties on response to IAPT treatment for depression and anxiety. Behaviour Research and Therapy. 73, 1-7.

Grant, N., Hotopf, M., Breen, G., Cleare, A, M., Grey, N., Hepgul, N., King, S., Moran, P., Pariante, C, M., Wingrove, J., Young, A, H \& Tylee, A. (2014) Predicting outcome following psychological therapy in IAPT (PROMPT): a naturalistic project protocol. BMC Psychiatry. 14 (1) 170178.

Hepgul, N., King, S., Amarasinghe, M., Breen, G., Grant, N., Grey, N., Hotopf, M., Moran, P., Pariante, C, M., Tylee, A., Wingrove, J., Young, A, H \& Cleare, A, 
J. (2016) Clinical characteristics of patients assessed within an Improving Access to Psychological Therapies (IAPT) service: results from a naturalistic cohort study (Predicting Outcome Following Psychological Therapy; PROMPT). BMC Psychiatry. 16:52 DOI 10.1186/s12888-016-0736-6.

Laporte, L., Paris, J., Bergevin, T., Fraser, R \& Cardin, J, F. (2018) Clinical outcomes of a stepped care programme for borderline personality disorder. Personality and Mental Health. 12, 252-264.

Livesley, W, J., Lang, K, L \& Vernon, P, A. (1998) Phenotypic and Genetic Structure of Traits Delineating Personality Disorder. Archive of General Psychiatry. 55 941-948.

Livesley, W. J. (2005) Principles and strategies for treating personality disorder. Canadian Journal of Psychiatry. 50, 442-450.

Mind. (2018) Shining Lights in Dark Corners of People's Lives; The Consensus Statement for People with Complex Mental Health Difficulties who are diagnosed with a Personality Disorder. https://www.mind.org.uk/media/21163353/consensusstatement-final.pdf (Last Accessed 16/08/218).

Moran, P. \& Crawford, M. J. (2013) Assessing the severity of borderline personality disorder. British Journal of Psychiatry. 203, 163-164.

Moran, P., Leese, M., Lee, T., Walters, P., Thormicroft, G \& Mann, A. (2003) Standardised Assessment of Personality - Abbreviated Scale (SAPAS): Preliminary validation of a brief screen for personality disorder. British Journal of Psychiatry. 183, 228-232.

National Institute for Health and Clinical Excellence (NICE). (2009) Borderline Personality Disorder: Treatment and Management. www.nice.org.uk (Last accessed:16/08/2018).

Paris, J. (2013) Stepped care: An alternative to routine extended treatment for patients with borderline personality disorder. Psychiatric Services. 64, 1035-1037. 
Paris, J. (2017) Stepped Care for Borderline Personality Disorder, Making Treatment Brief, Effective, and Accessible. Academic Press; London.

Public Health England. (2019) Public Health Profiles. https://fingertips.phe.org.uk/search/ethnicity\#pat/6/ati/102/par/E12000002 (Last accessed on 24/05/2019).

Ritchie, J., Lewis, J., McNaughton-Nicholls, C and Ormston, R. (2014) Qualitative Research Practice, A Guide for Social Science Students and Researchers (2nd ed.). London: Sage.

Richards, D, A., Bowe, P., Pagel, C., Weaver, A., Utley, M., Cape, J., Pilling, S., Lovell, K., Gilbody, S., Leibowitz, J., Owens, L., Paxton, R., Hennessy, S., Simpson, A., Gallivan, S., Tomson, D and Vasilakism C. (2012) Delivering stepped care: an analysis of implementation in routine practice. Implementation Science. 7:3 http://www.implementationscience.com/content/7/1/3 (Last accessed: 14th June 2019).

Royal College of Psychiatrists (2020) PS01/20: Services for people diagnosed with personality disorder. https://www.rcpsych.ac.uk/docs/default-source/improvingcare/better-mh-policy/position-statements/ps01 20.pdf?sfvrsn=85af7fbc 2 (last accessed: 29/05/2020)

Sampson, M, J., McCubbin, R, A \& Tyrer, P. (2006) Personality Disorder and Community Mental Health Teams, A Practitioner Guide. Wiley: Chichester.

Sweeney, A., Clement, S., Filson, B., Kennedy, A. (2016) "Trauma-informed mental healthcare in the UK: what is it and how can we further its development?", Mental Health Review Journal. Vol. 21 Issue: 3, pp.174-192.

Tyrer, P and Duggan, C. (2007) Nice Guidelines for the Treatment of Personality Disorder. Psychiatry. 7 (3) 109-111.

World Health Organisation. (2019) International Statistical Classification of Diseases and Related Health Problems. (1 $1{ }^{\text {th }}$ Revision) http://www.who.int/classifications/icd/en/ (Last accessed 29/05/20). 